DOI: $10.5216 /$ cab.v12i1.4823

\title{
ERITROLEUCOGRAMA DE EQUINOS SUBMETIDOS A OBSTRUÇÃO EXPERIMENTAL DO DUODENO, ÍLEO E CÓLON MAIOR
}

\author{
Paula Alessandra Di Filippo, ${ }^{1}$ Maria Augusta Berlingieri, ${ }^{2}$ Maristela de Cássia Seudo Lopes, ${ }^{3}$ \\ Eugênio de Campos Filho, ${ }^{4}$ Aureo Evangelista Santana ${ }^{5}$ e Gener Tadeu Pereira ${ }^{6}$ \\ 1. Professora doutora do Laboratório de Clínicas e Cirurgia Animal, UENF, Campos dos Goytacazes, RJ. \\ E-mail: paula_difilippo@yahoo.com.br \\ 2. Pós-graduanda do Departamento de Clínica e Cirurgia Veterinária da FCAV, Unesp, Jaboticabal, SP \\ 3. Pós-graduanda do Departamento de Clínica e Cirurgia Veterinária da FCAV, Unesp, Jaboticabal, SP \\ 4. Técnico do Laboratório de Patologia Clínica, FCAV, Unesp \\ 5. Professor doutor do Departamento de Clínica e Cirurgia Veterinária da FCAV, Unesp, Jaboticabal, SP \\ 6. Professor doutor do Departamento de Ciências Exatas, FCAV, Unesp, Jaboticabal, SP.
}

\section{RESUMO}

Realizou-se este trabalho para avaliar e comparar as alterações físicas e no hemograma de equinos submetidos a um modelo experimental de obstrução intestinal. Para tanto, 24 animais foram distribuídos em quatro grupos: controle instrumentado (GI), obstrução do duodeno (GII), íleo (GIII) e cólon maior (GIV). As amostras de sangue foram colhidas e os exames físicos foram realizados antes da cirurgia (T0), durante as obstruções (T1i, T2i e T3i), e após as desobstruções (T3r, T24r, T72r, T120r e T168r). Os equinos do grupo GII, (nos T2i e T3i) e os do grupo GIII (nos T1i, T2i, T3i e T3r) apresentaram sinais de dor abdominal, atonia intestinal e aumento da frequência cardíaca e respiratória. Nesses mesmos períodos e grupos houve diminuição na contagem de leucócitos, a qual, posteriormente (T24r e T72r), cedeu lugar a leucocitose por neutrofilia, com desvio à esquerda. Diminuição gradativa e contínua na contagem dos leucócitos foi observada a partir do T120r, indicando, à semelhança das características clínicas, a resolução da disfunção orgânica associada a obstrução. Os resultados obtidos foram relacionados com lesão entérica, resultante do modelo de obstrução, e indicam que algumas alterações laboratoriais, quando analisadas em conjunto com os dados obtidos no exame físico, podem auxiliar na identificação do segmento intestinal obstruído, na elaboração do prognóstico e no acompanhamento da evolução do processo de cura.

PALAVRAS-CHAVES: Cavalo, hemograma, cólica, isquemia, reperfusão.

\section{ABSTRACT}

\section{ERYTHRO-LEUKOGRAM OF HORSES SUBMITTED TO EXPERIMENTAL OBSTRUCTION OF DUODENUM, ILEUM AND LARGE COLON}

This study aimed to evaluate and compare physical and hematological alterations in equines submitted to an experimental model of intestinal obstruction. Twenty-four animals were divided into four groups: instrumented control (GI), duodenum obstruction (GII), ileum obstruction (GIII), and large colon obstruction (GIV). Physical examination was carried out and blood samples were collected before surgery (T0), during the obstruction (T1i, T2 i and T3i), and after unblocking procedures (T3r, T24r, T72r, T120r and T168r). Animals from GII, at T2i and T3i, and animals from GIII, at T1i, T2i, T3i and T3r, presented signs of abdominal pain, reduced gastrointestinal sounds and increased heart and respiratory rates, as well as leukopenia and neutropenia, which later (T24r and T72r) developed to leukocytosis, neutrophilia and left shifts. From T120r up, gradual and continuous decrease of leucocytes counts was observed indicating, similarly to Clínical characteristics, the resolution of organic dysfunction associated to obstruction. The results were associated to enteric injury from the obstruction model 
showing that some laboratorial alterations when analyzed, including physical examination, can be helpful at identifying

KEYWORDS: Equine, hemogram, colic, ischemia, reperfusion.

\section{INTRODUÇÃO}

A síndrome cólica é uma das urgências mais frequentes na clínica de eqüinos. Apesar dos avanços em relação a métodos de diagnóstico, técnicas anestésicas e cirúrgicas e acompanhamento intensivo no pós-operatório, a mortalidade permanece alta. Considera-se que as alterações ocorridas nas alças intestinais favoreçam a transferência de bactérias e toxinas do lúmen intestinal para a corrente sanguínea, contribuindo para a manifestação de sinais sistêmicos diversos (MOORE \& BARTON, 2003).

Estudo realizado por FAGLIARI \& SILVA (2002) demonstrou que houve elevação da temperatura retal, das frequências cardíaca e respiratória, do volume globular, dos valores das proteínas plasmáticas, do número de hemácias e de leucócitos em equinos com cólica, antes e após a laparotomia para correção de torção, compactação e encarceramento nefroesplênico de cólon maior. Resultados semelhantes foram obtidos por MONTELLO et al. (2004) em equinos submetidos a laparotomia em estação e enterotomia do cólon menor. Entretanto, segundo esses autores, as alterações hematológicas observadas, apesar de compatíveis com um quadro inflamatório agudo, não apresentaram correspondência clínica. Ademais, SVENDSEN et al. (1979) observaram que os animais que foram a óbito ou foram eutanasiados apresentaram alterações clínicas e laboratoriais mais acentuadas. Por fim, FAGLIARI et al. (2008), avaliando equinos com cólica que vieram a óbito de sete a dez dias após a cirurgia com sinais de choque séptico concluíram que, dentre os exames laboratoriais considerados indicadores de prognóstico para abdômen agudo de equinos, destacam-se as contagens de neutrófilos segmentados e de bastonetes.

Diante dessas observações, pelo presente estudo objetivou-se avaliar e comparar as alterações no eritroleucograma e no exame físico de equinos submetidos a obstrução experimental do duodeno, íleo e cólon maior. Buscou-se determinar o início e o comportamento, em função do tempo, de tais alterações e se estas podem ser which intestinal segment is blocked, drawing up the prognosis and following the evolution of the healing process.

utilizadas no diagnóstico e prognóstico das obstruções intestinais específicas em equinos com cólica.

\section{MATERIAL E MÉTODOS}

Utilizaram-se 24 equinos, sendo oito fêmeas (não gestantes) e dezesseis machos (doze castrados e quatro inteiros), sem raça definida, com média de idade de 6,2 $\pm 3,0$ anos, escore corporal de três a quatro (SPEIRS, 1997) e peso corporal médio de 295,9 \pm $32,7 \mathrm{~kg}$. Uma semana antes do experimento fez-se o controle de endoparasitas (mebendazol, ${ }^{1} 50 \mathrm{mg} \mathrm{kg}^{-1}$ ) e de ectoparasitas (deltametrina ${ }^{2}$ a $0,025 \%$ ). Os animais foram alojados em piquetes coletivos com dieta à base de feno de Coast cross (Cynodon dactylon) e água à vontade. Forneceu-se a ração concentrada comercial $^{3}$ duas vezes ao dia, em quantidade equivalente a $1 \%$ do peso corpóreo $(2,5$ a $3,4 \mathrm{~kg})$, adicionada de $50 \mathrm{~g} / \mathrm{dia}$ de suplemento mineral. ${ }^{4}$

Os equinos foram separados em quatro grupos de seis animais (duas fêmeas, três machos castrados e um inteiro): um grupo controle instrumentado (G1) sem realização da obstrução intestinal, porém, submetido aos mesmos procedimentos anestésicos e cirúrgicos descritos para os animais dos demais grupos e três grupos obstruídos. A separação dos grupos deu-se de modo uniforme em relação à idade, ao sexo e ao escore corporal. Realizaram-se as obstruções intestinais em três diferentes segmentos: duodeno (GII), íleo (GIII) e cólon maior - flexura pélvica (GIV).

Os animais foram contidos em brete e após tricotomia e antissepsia da fossa paralombar foram sedados com acepromazina $1 \%{ }^{5}\left(0,025 \mathrm{mg} \mathrm{kg}^{-1}, \mathrm{IV}\right)$, cloridrato de xilazina $2 \%{ }^{6}\left(0,5 \mathrm{mg} \mathrm{kg}^{-1}, \mathrm{IV}\right)$ e meperidina ${ }^{7}(4 \mathrm{mg}$ $\left.\mathrm{kg}^{-1}, \mathrm{IM}\right)$. Ato contínuo procedeu-se à anestesia local

\footnotetext{
Platelmin Equino - UCB S.A.

2 Butox P - Intervet S.A.

3 Tec Horse - Purina.

4 Omolen Ephos - Purina.

5 Acepran 1\% - Univet S.A.

6 Virbaxil 2\% - Virbac.

7 Dolosal-Cristália.
} 
infiltrativa, utilizando-se uma associação (1:1) de lidocaína $2 \%^{8}$ e de bupivacaína $0,75 \%{ }^{9}$, ambas sem vasoconstritor. Para mimetizar ao máximo as condições naturais, os animais ensaiados não foram submetidos a jejum hídrico e alimentar prévios.

Com os animais em posição quadrupedal, por meio da laparotomia - flanco direito para duodeno e íleo, e esquerdo para cólon maior -, os segmentos intestinais foram identificados. Em seguida, um dreno de Penrose $n^{\circ} 3$ foi posicionado ao redor da alça intestinal e, após o seu fechamento, iniciou-se a obstrução intestinal, segundo modelo descrito por DATT \& USENIK (1975). Nesse momento os animais receberam $1,5 \mathrm{mg} \mathrm{kg}^{-1}$, IV de cloridrato de tramadol ${ }^{10}$ que, diferentemente dos demais analgésicos opioides, não produz alterações hematológicas, cardiovasculares ou respiratórias relevantes e não afeta a motilidade ou a atividade metabólica intestinal (NATALINI \& ROBINSON, 2000). Sequencialmente, procedeu-se à sutura simples contínua dos músculos transversos do abdômen e da pele, utilizando-se vicryl $\mathrm{n}^{\circ}$ 2-0 e náilon $\mathrm{n}^{\circ} 4$, respectivamente. As obstruções foram mantidas por três horas; após esse período, promoveu-se a reversão das obstruções, tendo como acesso cirúrgico e protocolo os mesmos utilizados para promovê-las. Os drenos foram então removidos e as cavidades abdominais fechadas de acordo com a técnica descrita por TURNER \& McILWRAITH (2002).

No pós-operatório foi instituída terapia antimicrobiana com penicilina benzatina, ${ }^{11}$ na dose de 30.000 UI kg-1 IM, a cada 48 horas, perfazendo três aplicações. Como analgésico e anti-inflamatório administrou-se flunixim meglumine, ${ }^{12}$ na dose de $0,5 \mathrm{mg} \mathrm{kg}^{-1}, \mathrm{IV}$, a cada 24 horas, durante dois dias. Foi realizado curativo da ferida cirúrgica com polivinilpirrolidona-iodo tópica a $1 \%$, duas vezes ao dia, até a retirada dos pontos, no décimo dia após o procedimento.

Todos os animais foram submetidos ao exame físico e as características avaliadas incluíram temperatura retal $\left({ }^{\circ} \mathrm{C}\right)$, frequência cardíaca (bpm) e respiratória (mpm), tempo de preenchimento capilar (seg), coloração das membranas mucosas gengivais (0-1),

8 Lidovet-Bravet.

9 Neocaína $0,75 \%$ - Cristália.

10 Tramal - Cristália.

11 Pentabiótico veterinário reforçado - Fort Dodge.

12 Flunixina injetável - UCB S.A. auscultação abdominal (0-3), grau de dor abdominal (0-3) e refluxo gastrintestinal (0-1).

Para a realização do eritroleucograma foram utilizadas amostras de sangue obtidas mediante punção da jugular. As amostras foram acondicionadas em frascos contendo ácido etileno diamino tetracético (EDTA). Ato contínuo, as amostras de sangue foram processadas, obtendo-se o número de eritrócitos, a concentração de hemoglobina, o volume globular e a contagem global de leucócitos com o auxílio de um contador automático de células. ${ }^{13}$ Realizou-se em seguida a contagem diferencial de leucócitos em esfregaço corado pelo método de Giemsa, segundo JAIN (1993). A concentração plasmática de proteína total foi mensurada por refratometria.

Para cada equino as amostras de sangue e os exames físicos foram realizados antes do início do procedimento de obstrução (T0 ou basal); uma (T1i), duas (T2i); e três (T3i) horas após a realização da obstrução intestinal (fase de isquemia) e três (T3r), 24 (T24r), 72 (T72r), 120 (T120r) e 168 (T168r) horas após a desobstrução (fase de reperfusão).

Utilizou-se um delineamento experimental inteiramente casualizado, com quatro grupos e nove colheitas. Quando se constatou significância entre os grupos, em cada momento do estudo, aplicou-se o teste de Tukey para comparação das médias (SAMPAIO, 2002), através do programa estatístico SAS. ${ }^{14}$ Para as variáveis não paramétricas (coloração das membranas mucosas, auscultação abdominal, grau de dor abdominal e refluxo gástrico) foram utilizados os testes Kruskal-Wallis ou Wilcoxon. Todos os resultados foram considerados significativos quando $\mathrm{P}<0,05$.

\section{RESULTADOS E DISCUSSÃO}

Os valores relativos às frequências cardíaca e respiratória, ao tempo de preenchimento capilar e à temperatura retal, com as respectivas médias, desvios padrão e estatística calculada, estão expressos na Tabela 1.

13 Vet $\mathrm{ABC}^{\mathrm{TM}}$ - Animal Blood Counter. Horiba ABEX - Montpelier, made in France; série: 609AB11224.

14 Statistical Analysis of System - versão 8. 
TABELA 1. Média e desvio padrão da frequência cardíaca, frequência respiratória, tempo de perfusão periférica e temperatura retal dos equinos dos grupos I, II, III e IV, avaliados em diferentes momentos durante isquemia e reperfusão intestinal.

\begin{tabular}{|c|c|c|c|c|c|c|c|c|c|}
\hline \multicolumn{2}{|c|}{ Grupos } & \multicolumn{8}{|c|}{ Tempo (horas) } \\
\hline$(n=6)$ & T0 & T1i & $\mathrm{T} 2 \mathrm{i}$ & T3i & $\mathrm{T} 3 \mathrm{r}$ & $\mathrm{T} 24 \mathrm{r}$ & $\mathrm{T} 72 \mathrm{r}$ & $\mathrm{T} 120 \mathrm{r}$ & $\mathrm{T} 168 \mathrm{r}$ \\
\hline \multicolumn{10}{|c|}{ Frequência cardíaca (bpm) } \\
\hline I & $\begin{array}{c}35,66 \pm 2,33 \\
\text { A }\end{array}$ & $\begin{array}{c}37,00 \pm 6,06 \\
\text { B }\end{array}$ & $\begin{array}{c}39,33 \pm 6,28 \\
\text { B }\end{array}$ & $\begin{array}{c}37,66 \pm 1,96 \\
\text { B }\end{array}$ & $\begin{array}{c}42,66 \pm 8,11 \\
\text { B }\end{array}$ & $\begin{array}{c}36,66 \pm 3,72 \\
\text { A }\end{array}$ & $\begin{array}{c}41,33 \pm 4,88 \\
\text { A }\end{array}$ & $\begin{array}{c}38,33 \pm 4,63 \\
\mathrm{~A}\end{array}$ & $\begin{array}{c}37,33 \pm 5,46 \\
\text { A }\end{array}$ \\
\hline \multirow{2}{*}{ II } & $30,83 \pm 5,74$ & $41,00 \pm 4,73$ & $49,66 \pm 1,86$ & $50,16 \pm 2,04$ & $47,00 \pm 20,34$ & $41,00 \pm 7,97$ & $42,66 \pm 9,20$ & $39,00 \pm 8,07$ & $39,33 \pm 5,88$ \\
\hline & $\mathrm{A}$ & $\mathrm{AB}$ & A & $\mathrm{A}$ & $\mathrm{AB}$ & A & $\mathrm{A}$ & A & A \\
\hline \multirow{2}{*}{ III } & $31,83 \pm 5,34$ & $49,33 \pm 4,27$ & $48,50 \pm 5,78$ & $46,16 \pm 7,38$ & $57,66 \pm 7,42$ & $42,66 \pm 5,00$ & $45,50 \pm 9,95$ & $42,00 \pm 5,21$ & $40,00 \pm 4,19$ \\
\hline & & & A & A & A & A & A & A & A \\
\hline \multirow{2}{*}{ IV } & $37,00 \pm 7,34$ & $41,83 \pm 6,46$ & $40,83 \pm 5,74$ & $41,66 \pm 3,61$ & $44,33 \pm 11,82$ & $42,00 \pm 8,17$ & $45,50 \pm 5,78$ & $43,00 \pm 5,76$ & $45,83 \pm 6,08$ \\
\hline & A & $\mathrm{AB}$ & $\mathrm{B}$ & $\mathrm{B}$ & $\mathrm{AB}$ & $\mathrm{A}$ & A & $\mathrm{A}$ & A \\
\hline \multicolumn{10}{|c|}{ Frequência respiratória (mpm) } \\
\hline \multirow{2}{*}{ I } & $9,66 \pm 5,31$ & $9,33 \pm 2,06$ & $9,33 \pm 2,06$ & $9,50 \pm 2,50$ & $8,66 \pm 2,73$ & $8,00 \pm 2,19$ & $9,00 \pm 2,44$ & $11,33 \pm 1,03$ & $13,33 \pm 2,06$ \\
\hline & A & $\mathrm{B}$ & $\mathrm{B}$ & $\mathrm{B}$ & $\mathrm{B}$ & A & A & A & A \\
\hline \multirow{2}{*}{ II } & $10,00 \pm 2,82$ & $11,66 \pm 5,71$ & $15,33 \pm 3,93$ & $16,00 \pm 5,05$ & $10,83 \pm 2,40$ & $11,33 \pm 2,06$ & $12,33 \pm 2,33$ & $11,33 \pm 3,01$ & $12,33 \pm 3,66$ \\
\hline & A & $\mathrm{AB}$ & A & A & B & A & A & A & A \\
\hline \multirow{2}{*}{ III } & $10,66 \pm 3,72$ & $14,00 \pm 2,00$ & $15,66 \pm 2,65$ & $16,16 \pm 2,56$ & $15,83 \pm 5,23$ & $10,00 \pm 2,82$ & $11,83 \pm 3,12$ & $10,00 \pm 1,78$ & $11,66 \pm 2,65$ \\
\hline & A & A & A & A & A & A & A & A & $\mathrm{A}$ \\
\hline \multirow{2}{*}{ IV } & $10,66 \pm 1,63$ & $11,00 \pm 2,44$ & $12,00 \pm 2,52$ & $12,00 \pm 2,52$ & $11,66 \pm 2,65$ & $11,66 \pm 2,65$ & $11,00 \pm 6,16$ & $12,66 \pm 1,63$ & $12,00 \pm 2,52$ \\
\hline & A & $\mathrm{AB}$ & $\mathrm{AB}$ & $\mathrm{AB}$ & $\mathrm{AB}$ & A & $\mathrm{A}$ & A & A \\
\hline \multicolumn{10}{|c|}{ Tempo de perfusão periférica (seg) } \\
\hline \multirow[t]{2}{*}{ I } & $2,00 \pm 0,00$ & $2,00 \pm 0,00$ & $2,00 \pm 0,00$ & $2,00 \pm 0,00$ & $2,00 \pm 0,00$ & $2,00 \pm 0,00$ & $2,00 \pm 0,00$ & $2,00 \pm 0,00$ & $2,00 \pm 0,00$ \\
\hline & A & A & $\mathrm{B}$ & $\mathrm{B}$ & A & A & A & A & A \\
\hline \multirow{2}{*}{ II } & $2,00 \pm 0,00$ & $2,00 \pm 0,00$ & $2,16 \pm 0,40$ & $2,16 \pm 0,40$ & $2,00 \pm 0,00$ & $2,00 \pm 0,00$ & $2,00 \pm 0,00$ & $2,00 \pm 0,00$ & $2,00 \pm 0,00$ \\
\hline & A & A & A & A & A & A & A & A & A \\
\hline \multirow{2}{*}{ III } & $2,00 \pm 0,00$ & $2,00 \pm 0,00$ & $2,00 \pm 0,00$ & $2,00 \pm 0,00$ & $2,00 \pm 0,00$ & $2,00 \pm 0,00$ & $2,00 \pm 0,00$ & $2,00 \pm 0,00$ & $2,00 \pm 0,00$ \\
\hline & A & A & $\mathrm{B}$ & $\mathrm{B}$ & A & A & A & A & A \\
\hline \multirow{2}{*}{ IV } & $2,00 \pm 0,00$ & $2,00 \pm 0,00$ & $2,00 \pm 0,00$ & $2,00 \pm 0,00$ & $2,00 \pm 0,00$ & $2,00 \pm 0,00$ & $2,00 \pm 0,00$ & $2,00 \pm 0,00$ & $2,00 \pm 0,00$ \\
\hline & A & A & $\mathrm{B}$ & $\mathrm{B}$ & A & A & A & A & A \\
\hline \multicolumn{10}{|c|}{ Temperatura retal $\left({ }^{\circ} \mathrm{C}\right)$} \\
\hline & $37,40 \pm 0,99$ & $36,63 \pm 0,33$ & $37,06 \pm 0,27$ & $37,33 \pm 0,36$ & $37,93 \pm 0,13$ & $37,66 \pm 2,19$ & $37,50 \pm 0,57$ & $37,88 \pm 0,54$ & $36,88 \pm 0,33$ \\
\hline & $\mathrm{A}$ & A & A & A & A & A & A & $\mathrm{A}$ & $\mathrm{A}$ \\
\hline \multirow{2}{*}{ II } & $37,25 \pm 0,95$ & $37,25 \pm 0,71$ & $36,98 \pm 0,63$ & $37,25 \pm 0,81$ & $37,28 \pm 1,27$ & $37,95 \pm 0,65$ & $37,66 \pm 0,95$ & $37,63 \pm 0,71$ & $37,65 \pm 1,05$ \\
\hline & A & A & A & A & A & A & $\mathrm{A}$ & A & A \\
\hline \multirow{2}{*}{ III } & $36,75 \pm 0,37$ & $36,98 \pm 0,47$ & $37,01 \pm 0,65$ & $36,91 \pm 0,54$ & $37,26 \pm 0,79$ & $37,81 \pm 0,82$ & $37,41 \pm 0,29$ & $37,40 \pm 0,68$ & $36,91 \pm 0,41$ \\
\hline & A & A & A & $\mathrm{A}$ & A & A & A & A & A \\
\hline \multirow{2}{*}{ IV } & $36,91 \pm 0,64$ & $36,88 \pm 0,49$ & $36,81 \pm 0,58$ & $37,08 \pm 0,35$ & $37,63 \pm 0,38$ & $37,66 \pm 0,30$ & $37,63 \pm 0,32$ & $37,93 \pm 0,29$ & $37,76 \pm 0,43$ \\
\hline & A & $\mathrm{A}$ & $\mathrm{A}$ & A & A & A & A & A & A \\
\hline
\end{tabular}

T0: basal; T1i-T3i: horas correspondentes à fase de isquemia; T3r-T168r: horas correspondentes à fase de reperfusão; $\mathrm{I}=$ grupo controle (GI); II = grupo de animais submetidos a isquemia e reperfusão do duodeno (GII); III = animais submetidos a isquemia e reperfusão do íleo (GIII); IV = animais submetidos a isquemia e reperfusão do cólon maior (GIV).

Médias seguidas por letras distintas, na mesma coluna, diferem entre si pelo teste de Tukey, no nível de significância de 5\%, e estabelecem comparação entre os diferentes grupos em cada momento.

Durante todo o período experimental não foram observadas alterações nos valores de temperatura retal. Esses resultados diferem dos de SVENDSEN et al. (1979), FAGLIARI \& SILVA (2002) e FAGLIARI et al. (2008), para os quais a hipertermia deveu-se, possivelmente, à liberação de mediadores inflamatórios em decorrência da própria afecção intestinal e do trauma cirúrgico.

Os equinos do grupo GII, nos T2i e T3i e os do grupo GIII, nos T1i, T2i, T3i e T3r apresentaram aumento nos valores das frequências cardíaca e respiratória. Esse aumento possivelmente foi mediado pelo sistema nervoso autônomo, como resposta à dor ocasionada pela distensão das alças intestinais por digesta e gás, como descrito por MOORE \& BARTON (2003) e por SOUTHWOOD (2006). Nos animais do grupo GII, a distensão gástrica (Tabela 3) pode ter contribuído para as alterações cardiorespiratórias. Esse resultado corrobora o do experimento de cinco dos seis animais submetidos em que a obstrução experimental 
do duodeno DATT \& USENIK (1975) foram a óbito por ruptura gástrica. Segundo esses autores, a distensão gástrica deveu-se à pequena extensão intestinal cranial, à obstrução, ao conteúdo gástrico e à contínua capacidade secretora do estômago.

Neste ensaio, nos animais do grupo GII, a quantidade de fluido obtido através de sonda nasoesofágica foi 1 e 1,5 litros, contendo principalmente partículas alimentares, de coloração verde, odor fermentado, e com $\mathrm{pH}$ entre 4 e 5 . O refluxo gástrico pode ter contribuído para as discretas alterações nos valores do tempo de perfusão periférica e na coloração das membranas mucosas apresentadas pelos animais do GII, nos T2i e T3i (Tabelas 1 e 3). Entretanto, diferindo dos resultados obtidos por PUOTUNEN-REINERT \& HUSKAMP (1986), a perda de líquido resultante do modelo de obstrução não acarretou alterações nos valores do eritrograma (Tabela 2), provavelmente em função do menor tempo de manutenção das obstruções, como explicaram SVENDSEN et al. (1979).

TABELA 2. Média e desvio padrão da contagem de hemácias, concentração de hemoglobina, volume globular e da concentração das proteínas plasmáticas totais no sangue dos equinos dos grupos I, II, III e IV, avaliados em diferentes momentos durante isquemia e reperfusão intestinal

\begin{tabular}{|c|c|c|c|c|c|c|c|c|c|}
\hline \multirow{2}{*}{$\begin{array}{l}\text { Grupos } \\
(\mathrm{n}=6)\end{array}$} & \multirow[b]{2}{*}{ T0 } & \multicolumn{8}{|c|}{ Tempo (horas) } \\
\hline & & T1i & $\mathrm{T} 2 \mathrm{i}$ & T3i & $\mathrm{T} 3 \mathrm{r}$ & $\mathrm{T} 24 \mathrm{r}$ & $\mathrm{T} 72 \mathrm{r}$ & T120r & T168r \\
\hline \multicolumn{10}{|c|}{ Hemácias $\left(\times 10^{6} / \mu \mathrm{L}\right)$} \\
\hline I & $\begin{array}{c}5,53 \pm 1,00 \\
\mathrm{~A}\end{array}$ & $\begin{array}{c}5,08 \pm 1,05 \\
\text { A }\end{array}$ & $\begin{array}{c}5,22 \pm 1,12 \\
\text { A }\end{array}$ & $\begin{array}{c}5,08 \pm 1,10 \\
\mathrm{~A}\end{array}$ & $\begin{array}{c}5,23 \pm 1,00 \\
\mathrm{~A}\end{array}$ & $\begin{array}{c}5,50 \pm 0,87 \\
\mathrm{~A}\end{array}$ & $\begin{array}{c}5,62 \pm 0,66 \\
\text { A }\end{array}$ & $\begin{array}{c}5,57 \pm 0,48 \\
\text { A }\end{array}$ & $\begin{array}{c}5,75 \pm 0,53 \\
\text { A }\end{array}$ \\
\hline II & $\begin{array}{c}6,81 \pm 1,29 \\
\text { A }\end{array}$ & $\begin{array}{c}5,46 \pm 1,17 \\
\mathrm{~A}\end{array}$ & $\begin{array}{c}5,76 \pm 1,20 \\
\mathrm{~A}\end{array}$ & $\begin{array}{c}6,35 \pm 1,87 \\
\mathrm{~A}\end{array}$ & $\begin{array}{c}5,52 \pm 1,38 \\
\mathrm{~A}\end{array}$ & $\begin{array}{c}5,92 \pm 1,12 \\
\mathrm{~A}\end{array}$ & $\begin{array}{c}6,43 \pm 1,13 \\
\mathrm{~A}\end{array}$ & $\begin{array}{c}6,25 \pm 1,51 \\
\mathrm{~A}\end{array}$ & $\begin{array}{c}5,82 \pm 0,88 \\
\text { A }\end{array}$ \\
\hline III & $\begin{array}{c}5,92 \pm 0,65 \\
\mathrm{~A}\end{array}$ & $\begin{array}{c}5,28 \pm 0,78 \\
\mathrm{~A}\end{array}$ & $\begin{array}{c}5,53 \pm 0,79 \\
\mathrm{~A}\end{array}$ & $\begin{array}{c}5,60 \pm 0,60 \\
\mathrm{~A}\end{array}$ & $\begin{array}{c}5,21 \pm 0,68 \\
\mathrm{~A}\end{array}$ & $\begin{array}{c}5,54 \pm 0,62 \\
\mathrm{~A}\end{array}$ & $\begin{array}{c}5,81 \pm 0,64 \\
\mathrm{~A}\end{array}$ & $\begin{array}{c}5,56 \pm 0,59 \\
\text { A }\end{array}$ & $\begin{array}{c}5,56 \pm 0,44 \\
\mathrm{~A}\end{array}$ \\
\hline IV & $\begin{array}{c}5,77 \pm 1,61 \\
\mathrm{~A}\end{array}$ & $\begin{array}{c}5,13 \pm 1,50 \\
\mathrm{~A}\end{array}$ & $\begin{array}{c}5,31 \pm 1,37 \\
\mathrm{~A}\end{array}$ & $\begin{array}{c}5,40 \pm 1,33 \\
\mathrm{~A}\end{array}$ & $\begin{array}{c}5,11 \pm 1,41 \\
\mathrm{~A}\end{array}$ & $\begin{array}{c}5,80 \pm 1,57 \\
\mathrm{~A}\end{array}$ & $\begin{array}{c}5,58 \pm 1,12 \\
\mathrm{~A}\end{array}$ & $\begin{array}{c}5,78 \pm 1,32 \\
\mathrm{~A}\end{array}$ & $\begin{array}{c}5,63 \pm 1,30 \\
\mathrm{~A}\end{array}$ \\
\hline \multicolumn{10}{|c|}{ Hemoglobina $(\mathrm{g} / \mathrm{dL})$} \\
\hline I & $\begin{array}{c}8,86 \pm 1,02 \\
\mathrm{~A}\end{array}$ & $\begin{array}{c}8,13 \pm 1,00 \\
\mathrm{~A}\end{array}$ & $\begin{array}{c}8,16 \pm 1,08 \\
\text { A }\end{array}$ & $\begin{array}{c}7,93 \pm 0,91 \\
\mathrm{~A}\end{array}$ & $\begin{array}{c}8,13 \pm 0,72 \\
\mathrm{~A}\end{array}$ & $\begin{array}{c}8,66 \pm 0,89 \\
\text { A }\end{array}$ & $\begin{array}{c}9,13 \pm 0,45 \\
\text { A }\end{array}$ & $\begin{array}{c}8,76 \pm 0,52 \\
\text { A }\end{array}$ & $\begin{array}{c}9,06 \pm 0,31 \\
\text { A }\end{array}$ \\
\hline II & $\begin{array}{c}10,38 \pm 1,33 \\
\mathrm{~A}\end{array}$ & $\begin{array}{c}8,35 \pm 1,12 \\
\mathrm{~A}\end{array}$ & $\begin{array}{c}8,71 \pm 1,21 \\
\mathrm{~A}\end{array}$ & $\begin{array}{c}9,35 \pm 1,77 \\
\mathrm{~A}\end{array}$ & $\begin{array}{c}8,28 \pm 1,36 \\
\mathrm{~A}\end{array}$ & $\begin{array}{c}8,91 \pm 1,05 \\
\mathrm{~A}\end{array}$ & $\begin{array}{c}9,73 \pm 1,14 \\
\mathrm{~A}\end{array}$ & $\begin{array}{c}9,56 \pm 1,86 \\
\mathrm{~A}\end{array}$ & $\begin{array}{c}8,85 \pm 0,73 \\
\mathrm{~A}\end{array}$ \\
\hline III & $\begin{array}{c}9,46 \pm 1,05 \\
\mathrm{~A}\end{array}$ & $\begin{array}{c}8,53 \pm 1,37 \\
\mathrm{~A}\end{array}$ & $\begin{array}{c}8,95 \pm 1,40 \\
\text { A }\end{array}$ & $\begin{array}{c}8,98 \pm 0,99 \\
\text { A }\end{array}$ & $\begin{array}{c}8,20 \pm 1,03 \\
\mathrm{~A}\end{array}$ & $\begin{array}{c}8,88 \pm 1,04 \\
\mathrm{~A}\end{array}$ & $\begin{array}{c}9,36 \pm 1,30 \\
\mathrm{~A}\end{array}$ & $\begin{array}{c}9,05 \pm 1,06 \\
\mathrm{~A}\end{array}$ & $\begin{array}{c}8,88 \pm 0,91 \\
\mathrm{~A}\end{array}$ \\
\hline IV & $\begin{array}{c}9,48 \pm 2,26 \\
\mathrm{~A}\end{array}$ & $\begin{array}{c}8,38 \pm 2,03 \\
\mathrm{~A} \\
\end{array}$ & $\begin{array}{c}8,70 \pm 1,82 \\
\mathrm{~A}\end{array}$ & $\begin{array}{c}8,90 \pm 1,80 \\
\mathrm{~A}\end{array}$ & $\begin{array}{c}8,10 \pm 1,92 \\
\mathrm{~A}\end{array}$ & $\begin{array}{c}9,35 \pm 2,23 \\
\mathrm{~A} \\
\end{array}$ & $\begin{array}{c}9,13 \pm 1,66 \\
\mathrm{~A}\end{array}$ & $\begin{array}{c}9,46 \pm 1,79 \\
\mathrm{~A} \\
\end{array}$ & $\begin{array}{c}9,03 \pm 1,68 \\
\mathrm{~A}\end{array}$ \\
\hline \multicolumn{10}{|c|}{ Volume globular (\%) } \\
\hline I & $\begin{array}{c}26,06 \pm 3,22 \\
\text { A }\end{array}$ & $\begin{array}{c}23,76 \pm 3,29 \\
\text { A }\end{array}$ & $\begin{array}{c}24,43 \pm 3,35 \\
\text { A }\end{array}$ & $\begin{array}{c}23,70 \pm 3,17 \\
\mathrm{~A}\end{array}$ & $\begin{array}{c}24,40 \pm 2,55 \\
\text { A }\end{array}$ & $\begin{array}{c}25,80 \pm 2,66 \\
\text { A }\end{array}$ & $\begin{array}{c}26,50 \pm 1,63 \\
\text { A }\end{array}$ & $\begin{array}{c}26,30 \pm 0,46 \\
\text { A }\end{array}$ & $\begin{array}{c}27,03 \pm 0,58 \\
\text { A }\end{array}$ \\
\hline II & $\begin{array}{c}30,61 \pm 3,96 \\
\mathrm{~A}\end{array}$ & $\begin{array}{c}24,26 \pm 3,12 \\
\mathrm{~A}\end{array}$ & $\begin{array}{c}25,68 \pm 3,34 \\
\mathrm{~A}\end{array}$ & $\begin{array}{c}28,30 \pm 5,90 \\
\mathrm{~A}\end{array}$ & $\begin{array}{c}24,58 \pm 4,01 \\
\mathrm{~A}\end{array}$ & $\begin{array}{c}26,50 \pm 2,65 \\
\mathrm{~A}\end{array}$ & $\begin{array}{c}28,76 \pm 3,11 \\
\mathrm{~A}\end{array}$ & $\begin{array}{c}27,81 \pm 4,49 \\
\mathrm{~A}\end{array}$ & $\begin{array}{c}26,01 \pm 2,22 \\
\mathrm{~A}\end{array}$ \\
\hline III & $\begin{array}{c}28,30 \pm 3,46 \\
\mathrm{~A}\end{array}$ & $\begin{array}{c}25,25 \pm 4,10 \\
\mathrm{~A}\end{array}$ & $\begin{array}{c}26,40 \pm 4,03 \\
\text { A }\end{array}$ & $\begin{array}{c}26,71 \pm 2,90 \\
\text { A }\end{array}$ & $\begin{array}{c}24,81 \pm 2,97 \\
\text { A }\end{array}$ & $\begin{array}{c}26,58 \pm 3,68 \\
\text { A }\end{array}$ & $\begin{array}{c}27,83 \pm 3,13 \\
\text { A }\end{array}$ & $\begin{array}{c}26,90 \pm 2,26 \\
\text { A }\end{array}$ & $\begin{array}{c}26,65 \pm 2,76 \\
\text { A }\end{array}$ \\
\hline IV & $\begin{array}{c}27,73 \pm 7,33 \\
\mathrm{~A} \\
\end{array}$ & $\begin{array}{c}24,45 \pm 6,74 \\
\mathrm{~A}\end{array}$ & $\begin{array}{c}25,36 \pm 6,10 \\
\mathrm{~A}\end{array}$ & $\begin{array}{c}25,80 \pm 5,94 \\
\mathrm{~A} \\
\end{array}$ & $\begin{array}{c}24,36 \pm 6,34 \\
\mathrm{~A}\end{array}$ & $\begin{array}{c}27,80 \pm 7,23 \\
\mathrm{~A}\end{array}$ & $\begin{array}{c}26,78 \pm 4,86 \\
\mathrm{~A}\end{array}$ & $\begin{array}{c}27,70 \pm 5,75 \\
\mathrm{~A}\end{array}$ & $\begin{array}{c}27,10 \pm 5,79 \\
\mathrm{~A}\end{array}$ \\
\hline \multicolumn{10}{|c|}{ Proteína plasmática total $(\mathrm{g} / \mathrm{dL})$} \\
\hline I & $\begin{array}{c}7,46 \pm 0,27 \\
\text { A }\end{array}$ & $\begin{array}{c}7,00 \pm 0,35 \\
\mathrm{~A}\end{array}$ & $\begin{array}{c}7,00 \pm 0,38 \\
\text { A }\end{array}$ & $\begin{array}{c}6,93 \pm 0,41 \\
\text { A }\end{array}$ & $\begin{array}{c}6,86 \pm 0,59 \\
\text { A }\end{array}$ & $\begin{array}{c}7,40 \pm 0,35 \\
\text { A }\end{array}$ & $\begin{array}{c}7,40 \pm 0,35 \\
\text { A }\end{array}$ & $\begin{array}{c}7,26 \pm 0,51 \\
\mathrm{~A}\end{array}$ & $\begin{array}{c}7,60 \pm 0,47 \\
\text { A }\end{array}$ \\
\hline II & $\begin{array}{c}7,03 \pm 0,57 \\
\mathrm{~A}\end{array}$ & $\begin{array}{c}6,48 \pm 0,42 \\
\mathrm{~A}\end{array}$ & $\begin{array}{c}6,70 \pm 0,48 \\
\mathrm{~A}\end{array}$ & $\begin{array}{c}6,78 \pm 0,36 \\
\mathrm{~A}\end{array}$ & $\begin{array}{c}6,53 \pm 0,43 \\
\mathrm{~A}\end{array}$ & $\begin{array}{c}6,93 \pm 0,55 \\
\mathrm{~A}\end{array}$ & $\begin{array}{c}7,16 \pm 0,10 \\
\mathrm{~A}\end{array}$ & $\begin{array}{c}7,08 \pm 0,49 \\
\mathrm{~A}\end{array}$ & $\begin{array}{c}6,93 \pm 0,16 \\
\mathrm{~A}\end{array}$ \\
\hline III & $\begin{array}{c}7,25 \pm 0,40 \\
\mathrm{~A}\end{array}$ & $\begin{array}{c}6,68 \pm 0,30 \\
\mathrm{~A}\end{array}$ & $\begin{array}{c}6,86 \pm 0,32 \\
\mathrm{~A}\end{array}$ & $\begin{array}{c}6,71 \pm 0,27 \\
\text { A }\end{array}$ & $\begin{array}{c}6,58 \pm 0,22 \\
\mathrm{~A}\end{array}$ & $\begin{array}{c}7,30 \pm 0,51 \\
\mathrm{~A}\end{array}$ & $\begin{array}{c}7,35 \pm 0,08 \\
\mathrm{~A}\end{array}$ & $\begin{array}{c}7,20 \pm 0,17 \\
\text { A }\end{array}$ & $\begin{array}{c}7,35 \pm 0,28 \\
\mathrm{~A}\end{array}$ \\
\hline IV & $\begin{array}{c}7,00 \pm 0,82 \\
\mathrm{~A}\end{array}$ & $\begin{array}{c}6,68 \pm 0,72 \\
\mathrm{~A}\end{array}$ & $\begin{array}{c}6,86 \pm 0,70 \\
\mathrm{~A}\end{array}$ & $\begin{array}{c}6,88 \pm 0,85 \\
\mathrm{~A}\end{array}$ & $\begin{array}{c}6,78 \pm 0,92 \\
\mathrm{~A}\end{array}$ & $\begin{array}{c}7,20 \pm 0,99 \\
\mathrm{~A}\end{array}$ & $\begin{array}{c}6,96 \pm 0,71 \\
\mathrm{~A}\end{array}$ & $\begin{array}{c}7,10 \pm 0,81 \\
\mathrm{~A}\end{array}$ & $\begin{array}{c}7,13 \pm 0,72 \\
\mathrm{~A}\end{array}$ \\
\hline
\end{tabular}

T0: basal; T1i-T3i: horas correspondentes à fase de isquemia; T3r-T168r: horas correspondentes à fase de reperfusão ou de desobstrução; I = grupo controle (GI); II = grupo de animais submetidos a isquemia e reperfusão do duodeno (GII); III = animais submetidos a isquemia e reperfusão do íleo (GIII); IV = animais submetidos a isquemia e reperfusão do cólon maior (GIV).

Médias seguidas por letras distintas, na mesma coluna, diferem entre si pelo teste de Tukey, no nível de significância de 5\%, e estabelecem comparação entre os diferentes grupos em cada momento. 
A ausência de alterações no eritrograma e na concentração das proteínas plasmáticas totais (Tabela 2) demonstra que, independentemente de qual segmento intestinal foi obstruído, a manutenção das obstruções intestinais por três horas acarretou severa perda de fluidos e de elementos plasmáticos do compartimento intravascular para o interior do lúmen intestinal ou cavidade abdominal, como observado por SVENDSEN et al. (1979) e por DI FILIPPO et al. (2008).

DATT \& USENIK (1975) observaram elevações significativas do volume globular, da contagem de hemácias e das concentrações de hemoglobina e de proteínas plasmáticas totais em equinos submetidos a obstrução de duodeno e de íleo. Entretanto, as primeiras alterações somente foram observadas seis a doze horas após a realização das obstruções e, com exceção dos animais com obstrução de cólon menor, todos os demais foram a óbito.

Os sinais de dor abdominal apresentados pelos animais do grupo GII tiveram início a partir da segunda hora após a realização da obstrução, atingindo os maiores escores no T3i (terceira hora). Nos animais do grupo GIII, as alterações iniciaram-se já na primeirahora após a realização da obstrução (T1i) e permaneceram até as três horas de reperfusão (Tabela 3). Esses resultados foram compatíveis com as alterações nas frequências cardíaca e respiratória apresentadas pelos animais dos referidos grupos e tempos (Tabela 1) e deveram-se, possivelmente, à distensão das alças intestinais (SOUTHWOOD, 2006) e do estômago (DATT \& USENIK, 1975) por digesta e gás e pela liberação de mediadores inflamatórios, em decorrência da isquemia e reperfusão intestinal, como ressaltado por FALEIROS et al. (2001). Os sinais brandos e intermitentes apresentados pelos animais do GIV não foram associados a alterações clínicas e deveram-se ao maior diâmetro do segmento intestinal obstruído. A manutenção das obstruções intestinais por maior período de tempo desencadearia sinais de dor abdominal, tais quais os observados por DATT \& USENIK (1975) em modelo experimental similar. No referido ensaio, os animais submetidos a obstrução do cólon maior apresentaram sinais de dor abdominal intensa somente de 24 a 48 horas após a realização das obstruções.

Durante o período de obstrução e de desobstrução, principalmente nos T2i, T3i e T3r, os animais dos grupos GII e GIII apresentaram atonia intestinal (Ta- bela 3). Nesses mesmos períodos, nos animais do GIV, houve predomínio da hipomotilidade. Esses resultados foram associados à distensão das alças intestinais e também a possíveis desequilíbrios eletrolíticos, assim como citou SOUTHWOOD (2006).

$\mathrm{Na}$ Tabela 4, são apresentados os valores relativos à contagem global e diferencial de leucócitos, com as respectivas médias, desvios padrão e estatística calculada. Durante o período de obstrução (T1i a T3i), houve diminuição na contagem de leucócitos nos equinos dos grupos GII e GIII. Essa diminuição ainda se manteve, apesar de não ter sido estatisticamente significativa, durante a fase inicial de reperfusão (T3r). A redução foi ocasionada pela marginação e migração de neutrófilos para o foco inflamatório em resposta à lesão entérica, como explicaram SVENDSEN et al. (1979), JAIN (1993) e LASSEN \& SWARDSON (1995). Segundo BASILE-FILHO et al. (2002), a leucopenia é um achado normal durante a evolução da síndrome cólica, visto que os neutrófilos são os efetores fundamentais na defesa do organismo contra as infecções bacterianas, com o intuito de fagocitar microrganismos, células mortas e debris celulares. Entretanto, FAGLIARI \& SILVA (2002) observaram leucocitose por neutrofilia, com desvio à esquerda, desde a fase pré-cirúrgica em animais com cólica. Essas diferenças residem, segundo LASSEN \& SWARDSON (1995), no tempo de evolução do processo, difícil de ser mensurado em estudos casuísticos como o realizado por FAGLIARI \& SILVA (2002).

Somente no T3r houve acentuada diminuição no número de eosinófilos nos animais dos grupos GII e GIV (Tabela 4). A possível causa da eosinopenia foi a excitação, a dor e o estresse decorrentes da fase de reversão das obstruções. Segundo JAIN (1986), esses fatores desencadeiam a liberação de cortisol endógeno, o qual consequentemente induz à eosinopenia.

Nos T24r e T72r, nos animais do GII e do GIII, verificou-se à semelhança do observado por SVENDSEN et al. (1979), FAGLIARI \& SILVA (2002) e FAGLIARI et al. (2008) - leucocitose por neutrofilia com desvio à esquerda. Esse achado sinalizou para a presença de um processo inflamatório, resultante do modelo de obstrução, que por reter a digesta no lume intestinal predispôs à absorção de toxinas, o que foi confirmado pela presença de bastonetes (Tabela 4). Segundo LASSEN \& SWARDSON (1995), o desvio 
à esquerda é o indicador mais específico e sensível da presença de um processo inflamatório, e a magnitude do desvio indica o grau de demanda tecidual por neutrófilos.

Concomitantemente à leucocitose por neutrofilia observada nos T24r e T72r, os animais do GII e do GIII apresentaram diminuição do número de linfócitos. Esse resultado corrobora os de FAGLIARI \& SILVA (2002) e, segundo JAIN (1993), não é rara a ocorrência de lin- fopenia, em casos de neutrofilia, decorrente de infecção bacteriana ou toxemia, como acontece comumente em situações de abdômen agudo.

Diminuição gradativa e contínua na contagem dos leucócitos foi observada a partir do T120r, indicando, à semelhança das características clínicas, a resolução da disfunção orgânica associada à obstrução. Comentários semelhantes são válidos para as contagens de neutrófilos segmentados, neutrófilos bastonetes e linfócitos.

TABELA 3. Escores médios e desvio padrão para a coloração das membranas mucosas, refluxo gástrico, grau de dor e auscultação abdominal dos equinos dos grupos I, II, III e IV, avaliados em diferentes momentos durante isquemia e reperfusão intestinal

\begin{tabular}{|c|c|c|c|c|c|c|c|c|c|}
\hline \multirow{2}{*}{$\begin{array}{l}\text { Grupos } \\
(\mathrm{n}=6)\end{array}$} & \multirow[b]{2}{*}{ T0 } & \multicolumn{8}{|c|}{ Tempo (horas) } \\
\hline & & T1i & $\mathrm{T} 2 \mathrm{i}$ & T3i & $\mathrm{T} 3 \mathrm{r}$ & $\mathrm{T} 24 \mathrm{r}$ & $\mathrm{T} 72 \mathrm{r}$ & T120r & T168r \\
\hline \multicolumn{10}{|c|}{ Coloração das membranas mucosas } \\
\hline \multirow{2}{*}{ I } & $1,00 \pm 0,00$ & $1,00 \pm 0,00$ & $1,00 \pm 0,00$ & $1,00 \pm 0,00$ & $1,00 \pm 0,00$ & $1,00 \pm 0,00$ & $1,00 \pm 0,00$ & $1,00 \pm 0,00$ & $1,00 \pm 0,00$ \\
\hline & A & A & B & B & A & A & A & A & A \\
\hline \multirow{2}{*}{ II } & $1,00 \pm 0,00$ & $1,00 \pm 0,00$ & $1,16 \pm 0,40$ & $1,82 \pm 0,54$ & $1,00 \pm 0,00$ & $1,00 \pm 0,00$ & $1,00 \pm 0,00$ & $1,00 \pm 0,00$ & $1,00 \pm 0,00$ \\
\hline & A & A & A & A & A & A & A & A & A \\
\hline \multirow{2}{*}{ III } & $1,00 \pm 0,00$ & $1,00 \pm 0,00$ & $1,00 \pm 0,00$ & $1,00 \pm 0,00$ & $1,00 \pm 0,00$ & $1,00 \pm 0,00$ & $1,00 \pm 0,00$ & $1,00 \pm 0,00$ & $1,00 \pm 0,00$ \\
\hline & $\mathrm{A}$ & $\mathrm{A}$ & B & B & A & A & $\mathrm{A}$ & A & $\mathrm{A}$ \\
\hline \multirow{2}{*}{ IV } & $1,00 \pm 0,00$ & $1,00 \pm 0,00$ & $1,00 \pm 0,00$ & $1,00 \pm 0,00$ & $1,00 \pm 0,00$ & $1,00 \pm 0,00$ & $1,00 \pm 0,00$ & $1,00 \pm 0,00$ & $1,00 \pm 0,00$ \\
\hline & A & A & $\mathrm{B}$ & $\mathrm{B}$ & A & A & A & A & A \\
\hline \multicolumn{10}{|c|}{ Refluxo gástrico } \\
\hline \multirow[b]{2}{*}{ I } & $0,00 \pm 0,00$ & $0,00 \pm 0,00$ & $0,00 \pm 0,00$ & $0,00 \pm 0,00$ & $0,00 \pm 0,00$ & $0,00 \pm 0,00$ & $0,00 \pm 0,00$ & $0,00 \pm 0,00$ & $0,00 \pm 0,00$ \\
\hline & A & A & B & B & A & A & $\mathrm{A}$ & A & A \\
\hline \multirow{2}{*}{ II } & $0,00 \pm 0,00$ & $0,00 \pm 0,00$ & $0,50 \pm 0,54$ & $0,33 \pm 0,51$ & $0,00 \pm 0,00$ & $0,00 \pm 0,00$ & $0,00 \pm 0,00$ & $0,00 \pm 0,00$ & $0,00 \pm 0,00$ \\
\hline & A & A & A & A & A & A & A & A & A \\
\hline \multirow{2}{*}{ III } & $0,00 \pm 0,00$ & $0,00 \pm 0,00$ & $0,00 \pm 0,00$ & $0,00 \pm 0,00$ & $0,00 \pm 0,00$ & $0,00 \pm 0,00$ & $0,00 \pm 0,00$ & $0,00 \pm 0,00$ & $0,00 \pm 0,00$ \\
\hline & A & A & B & B & A & A & A & A & A \\
\hline \multirow{2}{*}{ IV } & $0,00 \pm 0,00$ & $0,00 \pm 0,00$ & $0,00 \pm 0,00$ & $0,00 \pm 0,00$ & $0,00 \pm 0,00$ & $0,00 \pm 0,00$ & $0,00 \pm 0,00$ & $0,00 \pm 0,00$ & $0,00 \pm 0,00$ \\
\hline & A & A & B & B & A & A & A & A & A \\
\hline \multicolumn{10}{|c|}{ Grau de dor } \\
\hline \multirow{2}{*}{ I } & $0,00 \pm 0,00$ & $0,00 \pm 0,00$ & $0,00 \pm 0,00$ & $0,10 \pm 0,02$ & $0,00 \pm 0,00$ & $0,00 \pm 0,00$ & $0,00 \pm 0,00$ & $0,00 \pm 0,00$ & $0,00 \pm 0,00$ \\
\hline & A & B & B & B & B & A & A & A & A \\
\hline \multirow{2}{*}{ II } & $0,00 \pm 0,00$ & $0,33 \pm 0,51$ & $1,66 \pm 1,03$ & $2,00 \pm 1,54$ & $0,50 \pm 0,83$ & $0,83 \pm 0,40$ & $0,50 \pm 0,54$ & $0,00 \pm 0,00$ & $0,00 \pm 0,00$ \\
\hline & A & B & A & A & $\mathrm{AB}$ & A & A & A & A \\
\hline \multirow{2}{*}{ III } & $0,00 \pm 0,00$ & $0,63 \pm 0,51$ & $2,33 \pm 0,81$ & $2,83 \pm 0,94$ & $1,50 \pm 0,54$ & $1,00 \pm 0,63$ & $0,40 \pm 0,32$ & $0,00 \pm 0,00$ & $0,00 \pm 0,00$ \\
\hline & A & A & A & A & A & A & A & A & A \\
\hline \multirow{2}{*}{ IV } & $0,00 \pm 0,00$ & $0,11 \pm 0,52$ & $0,33 \pm 0,51$ & $1,00 \pm 1,09$ & $0,36 \pm 0,51$ & $0,00 \pm 0,00$ & $0,00 \pm 0,00$ & $0,00 \pm 0,00$ & $0,00 \pm 0,00$ \\
\hline & A & $\mathrm{B}$ & $\mathrm{B}$ & $\mathrm{AB}$ & $\mathrm{B}$ & A & A & A & A \\
\hline \multicolumn{10}{|c|}{ Auscultação abdominal } \\
\hline \multirow{2}{*}{ I } & $2,00 \pm 0,00$ & $1,66 \pm 0,51$ & $2,00 \pm 0,00$ & $1,66 \pm 0,51$ & $1,66 \pm 0,51$ & $2,00 \pm 0,00$ & $2,00 \pm 0,00$ & $2,00 \pm 0,00$ & $2,00 \pm 0,00$ \\
\hline & A & A & A & A & A & A & A & A & A \\
\hline \multirow{2}{*}{ II } & $2,00 \pm 0,00$ & $1,33 \pm 0,51$ & $0,66 \pm 0,51$ & $0,50 \pm 0,54$ & $0,50 \pm 0,83$ & $0,83 \pm 0,40$ & $1,50 \pm 0,54$ & $2,00 \pm 0,00$ & $2,00 \pm 0,00$ \\
\hline & A & A & B & B & B & A & A & A & A \\
\hline \multirow{2}{*}{ III } & $2,00 \pm 0,00$ & $1,16 \pm 0,40$ & $0,33 \pm 0,51$ & $0,16 \pm 0,40$ & $0,83 \pm 0,40$ & $1,00 \pm 0,63$ & $2,00 \pm 0,00$ & $2,00 \pm 0,00$ & $2,00 \pm 0,00$ \\
\hline & A & A & B & B & $\mathrm{AB}$ & A & A & A & A \\
\hline \multirow{2}{*}{ IV } & $2,00 \pm 0,00$ & $2,00 \pm 0,00$ & $1,33 \pm 0,81$ & $1,33 \pm 1,03$ & $1,50 \pm 0,54$ & $2,00 \pm 0,00$ & $2,00 \pm 0,00$ & $2,00 \pm 0,00$ & $2,00 \pm 0,00$ \\
\hline & A & A & A & A & $\mathrm{AB}$ & A & A & A & A \\
\hline
\end{tabular}

T0: basal; T1i-T3i: horas correspondentes à fase de isquemia; T3r-T168r: horas correspondentes à fase de reperfusão ou de desobstrução; $\mathrm{I}=$ grupo controle (GI); II = grupo de animais submetidos a isquemia e reperfusão do duodeno (GII); III = animais submetidos a isquemia e reperfusão do íleo (GIII); IV = animais submetidos a isquemia e reperfusão do cólon maior (GIV).

Coloração das membranas mucosas: $1=$ normal; $2=$ hiperêmica; Refluxo gástrico: $0=$ ausente; $1=$ presente. Dor abdominal: $0=$ ausente; $1=$ leve (olhar para o flanco); 2 = moderada (cavar); $3=$ severa (deitar/rolar). Auscultação abdominal: $0=$ ausente; $1=$ diminuído; $2=$ normal; $3=$ aumentado (SPEIRS, 1997).

Médias seguidas por letras distintas, na mesma linha, diferem entre si pelo teste Kruskal-Wallis/Wilcoxon, no nível de significância de 5\%, e estabelecem comparação entre os diferentes grupos em cada momento. 
TABELA 4. Média e desvio padrão da contagem total de leucócitos, neutrófilos segmentados, bastonetes, linfócitos, monócitos, basófilos e eosinófilos no sangue dos equinos dos grupos I, II, III e IV, avaliados em diferentes momentos durante isquemia e reperfusão intestinal

\begin{tabular}{|c|c|c|c|c|c|c|c|c|c|}
\hline \multirow{2}{*}{$\frac{\text { Grupos }}{(\mathrm{n}=6)}$} & \multirow[b]{2}{*}{ T0 } & \multicolumn{8}{|c|}{ Tempo (horas) } \\
\hline & & T1i & $\mathrm{T} 2 \mathrm{i}$ & $\mathrm{T} 3 \mathrm{i}$ & $\mathrm{T} 3 \mathrm{r}$ & $\mathrm{T} 24 \mathrm{r}$ & $\mathrm{T} 72 \mathrm{r}$ & $120 \mathrm{r}$ & $168 \mathrm{r}$ \\
\hline \multicolumn{10}{|c|}{ Leucócitos $\left(\mathrm{x} 10^{3} / \mu \mathrm{L}\right)$} \\
\hline I & $\underset{\mathrm{A}}{9,93 \pm 1,96}$ & $\begin{array}{c}9,36 \pm 1,39 \\
\mathrm{~A}\end{array}$ & $\underset{\mathrm{A}}{8,93 \pm 1,59}$ & $\underset{\mathrm{A}}{8,93 \pm 1,51}$ & $\begin{array}{c}11,70 \pm 2,32 \\
\mathrm{~A}\end{array}$ & $\begin{array}{c}7,56 \pm 2,24 \\
\mathrm{~B}\end{array}$ & $\frac{8,76 \pm 2,30}{\mathrm{~B}}$ & $\underset{\mathrm{A}}{8,53 \pm 1,98}$ & $\frac{8,76 \pm 1,52}{A}$ \\
\hline II & $\underset{\mathrm{A}}{8,31 \pm 1,65}$ & $\begin{array}{c}7,16 \pm 3,14 \\
B\end{array}$ & $\begin{array}{c}7,58 \pm 2,84 \\
\mathrm{~B}\end{array}$ & $\begin{array}{c}7,48 \pm 2,60 \\
\mathrm{~B}\end{array}$ & $\begin{array}{c}8,53 \pm 3,92 \\
\mathrm{~A}\end{array}$ & $\begin{array}{c}13,3 \pm 2,98 \\
\mathrm{~A}\end{array}$ & $\begin{array}{c}13,04 \pm 1,61 \\
\mathrm{~A}\end{array}$ & $\begin{array}{c}9,60 \pm 2,88 \\
\mathrm{~A}\end{array}$ & $\begin{array}{c}7,66 \pm 2,62 \\
\mathrm{~A}\end{array}$ \\
\hline III & $\begin{array}{c}8,48 \pm 1,26 \\
\mathrm{~A}\end{array}$ & $\begin{array}{c}6,61 \pm 966 \\
B\end{array}$ & $\begin{array}{c}7,05 \pm 1,65 \\
\text { B }\end{array}$ & $\begin{array}{c}7,48 \pm 2,09 \\
\mathrm{~B}\end{array}$ & $\begin{array}{c}10,43 \pm 3,35 \\
\mathrm{~A}\end{array}$ & $\begin{array}{c}12,83 \pm 3,74 \\
\mathrm{~A}\end{array}$ & $\begin{array}{c}12,07 \pm 1,91 \\
\mathrm{~A}\end{array}$ & $\begin{array}{c}10,71 \pm 4,01 \\
\mathrm{~A}\end{array}$ & $\begin{array}{c}7,82 \pm 1,96 \\
\mathrm{~A}\end{array}$ \\
\hline IV & $\begin{array}{c}8,30 \pm 3,01 \\
\mathrm{~A}\end{array}$ & $\begin{array}{c}8,49 \pm 3,21 \\
\mathrm{AB}\end{array}$ & $\begin{array}{c}8,76 \pm 2,71 \\
\mathrm{AB}\end{array}$ & $\begin{array}{c}7,90 \pm 2,24 \\
\mathrm{AB}\end{array}$ & $\begin{array}{c}10,88 \pm 3,29 \\
\mathrm{~A}\end{array}$ & $\begin{array}{c}10,20 \pm 4,54 \\
\mathrm{AB}\end{array}$ & $\begin{array}{c}7,51 \pm 2,54 \\
\mathrm{~B}\end{array}$ & $\begin{array}{c}10,30 \pm 3,90 \\
\mathrm{~A}\end{array}$ & $\begin{array}{c}8,83 \pm 2,10 \\
\mathrm{~A}\end{array}$ \\
\hline \multicolumn{10}{|c|}{ Neutrófilo segmentado $\left(\mathrm{x} 10^{3} / \mu \mathrm{L}\right)$} \\
\hline I & $\begin{array}{c}6,42 \pm 1,75 \\
A\end{array}$ & $\begin{array}{c}5,87 \pm 1,54 \\
\text { B }\end{array}$ & $\begin{array}{c}5,57 \pm 1,51 \\
\mathrm{~A}\end{array}$ & $\begin{array}{c}5,98 \pm 1,29 \\
A\end{array}$ & $\begin{array}{c}8,91 \pm 1,56 \\
\mathrm{~A}\end{array}$ & $\begin{array}{c}4,77 \pm 1,86 \\
\mathrm{~B}\end{array}$ & $\begin{array}{c}4,90 \pm 2,28 \\
\mathrm{~B}\end{array}$ & $\begin{array}{c}5,94 \pm 1,99 \\
\mathrm{~A}\end{array}$ & $\begin{array}{c}6,19 \pm 2,00 \\
\mathrm{~A}\end{array}$ \\
\hline II & $\begin{array}{c}4,40 \pm 1,22 \\
\mathrm{~A}\end{array}$ & $\begin{array}{c}4,35 \pm 2,23 \\
\mathrm{AB}\end{array}$ & $\begin{array}{c}5,13 \pm 2,05 \\
\mathrm{~A}\end{array}$ & $\begin{array}{c}4,81 \pm 2,09 \\
\mathrm{~A}\end{array}$ & $\begin{array}{c}6,78 \pm 3,37 \\
\mathrm{~A}\end{array}$ & $\begin{array}{c}8,77 \pm 3,92 \\
\mathrm{~A}\end{array}$ & $\begin{array}{c}9,60 \pm 6,68 \\
\mathrm{~A}\end{array}$ & $\begin{array}{c}5,93 \pm 2,66 \\
\mathrm{~A}\end{array}$ & $\begin{array}{c}3,83 \pm 1,53 \\
\mathrm{~A}\end{array}$ \\
\hline III & $\begin{array}{c}4,69 \pm 1,23 \\
\mathrm{~A}\end{array}$ & $\begin{array}{c}3,99 \pm 1,13 \\
\mathrm{~A}\end{array}$ & $\begin{array}{c}4,87 \pm 1,85 \\
\mathrm{~A}\end{array}$ & $\begin{array}{c}5,08 \pm 2,50 \\
\mathrm{~A}\end{array}$ & $\begin{array}{c}8,44 \pm 3,45 \\
\text { A }\end{array}$ & $\begin{array}{c}10,56 \pm 3,81 \\
\mathrm{~A}\end{array}$ & $\begin{array}{c}9,14 \pm 1,16 \\
\mathrm{~A}\end{array}$ & $\begin{array}{c}7,21 \pm 3,76 \\
\mathrm{~A}\end{array}$ & $\begin{array}{c}4,95 \pm 1,75 \\
\mathrm{~A}\end{array}$ \\
\hline IV & $\begin{array}{c}4,56 \pm 1,98 \\
\mathrm{~A}\end{array}$ & $\begin{array}{c}5,69 \pm 2,21 \\
\mathrm{~B}\end{array}$ & $\begin{array}{c}5,57 \pm 1,74 \\
\mathrm{~A}\end{array}$ & $\begin{array}{c}4,99 \pm 1,51 \\
\mathrm{~A}\end{array}$ & $\begin{array}{c}8,73 \pm 2,75 \\
\mathrm{~A}\end{array}$ & $\begin{array}{c}7,80 \pm 3,74 \\
\mathrm{AB}\end{array}$ & $\begin{array}{c}4,34 \pm 1,89 \\
\mathrm{~B}\end{array}$ & $\begin{array}{c}7,13 \pm 3,86 \\
\mathrm{~A}\end{array}$ & $\begin{array}{c}5,33 \pm 1,66 \\
\mathrm{~A}\end{array}$ \\
\hline \multicolumn{10}{|c|}{ Neutrófilo bastonete $\left(\times 10^{3} / \mu \mathrm{L}\right)$} \\
\hline I & $\begin{array}{c}0,04 \pm 0,06 \\
\mathrm{~A}\end{array}$ & $\begin{array}{c}0,06 \pm 0,10 \\
\mathrm{~A}\end{array}$ & $\begin{array}{c}0,04 \pm 0,05 \\
\mathrm{~A}\end{array}$ & $\begin{array}{c}0,00 \pm 0,00 \\
\text { A }\end{array}$ & $\begin{array}{c}0,05 \pm 0,00 \\
\mathrm{~A}\end{array}$ & $\begin{array}{c}0,06 \pm 0,04 \\
\text { B }\end{array}$ & $\begin{array}{c}0,14 \pm 0,07 \\
\text { B }\end{array}$ & $\begin{array}{c}0,02 \pm 0,03 \\
\mathrm{~A}\end{array}$ & $\begin{array}{c}0,03 \pm 0,05 \\
\mathrm{~A}\end{array}$ \\
\hline II & $\begin{array}{c}0,02 \pm 0,04 \\
\mathrm{~A}\end{array}$ & $\begin{array}{c}0,02 \pm 0,02 \\
\mathrm{~A}\end{array}$ & $\begin{array}{c}0,03 \pm 0,05 \\
\mathrm{~A}\end{array}$ & $\begin{array}{c}0,01 \pm 0,02 \\
\mathrm{~A}\end{array}$ & $\begin{array}{c}0,09 \pm 0,11 \\
\mathrm{~A}\end{array}$ & $\begin{array}{c}0,35 \pm 0,042 \\
\mathrm{~A}\end{array}$ & $\begin{array}{c}0,43 \pm 0,17 \\
\text { A }\end{array}$ & $\begin{array}{c}0,07 \pm 0,10 \\
\text { A }\end{array}$ & $\begin{array}{c}0,11 \pm 0,05 \\
\mathrm{~A}\end{array}$ \\
\hline III & $\begin{array}{c}0,03 \pm 0,04 \\
\mathrm{~A}\end{array}$ & $\begin{array}{c}0,03 \pm 0,05 \\
\mathrm{~A}\end{array}$ & $\begin{array}{c}0,05 \pm 0,07 \\
\mathrm{~A}\end{array}$ & $\begin{array}{c}0,02 \pm 0,04 \\
\mathrm{~A}\end{array}$ & $\begin{array}{c}0,07 \pm 0,08 \\
\mathrm{~A}\end{array}$ & $\begin{array}{c}0,37 \pm 0,036 \\
\mathrm{~A}\end{array}$ & $\begin{array}{c}0,31 \pm 0,15 \\
\mathrm{AB}\end{array}$ & $\begin{array}{c}0,01 \pm 0,03 \\
\mathrm{~A}\end{array}$ & $\begin{array}{c}0,14 \pm 0,04 \\
\mathrm{~A}\end{array}$ \\
\hline $\mathrm{V}$ & $\begin{array}{c}0,03 \pm 0,03 \\
\mathrm{~A}\end{array}$ & $\begin{array}{c}0,04 \pm 0,05 \\
\mathrm{~A}\end{array}$ & $\begin{array}{c}0,03 \pm 0,02 \\
\mathrm{~A}\end{array}$ & $\begin{array}{c}0,01 \pm 0,03 \\
\mathrm{~A}\end{array}$ & $\begin{array}{c}0,08 \pm 0,05 \\
\mathrm{~A}\end{array}$ & $\begin{array}{c}0,10 \pm 0,09 \\
\mathrm{AB}\end{array}$ & $\begin{array}{c}0,04 \pm 0,10 \\
\mathrm{~B}\end{array}$ & $\begin{array}{c}0,05 \pm 0,12 \\
\mathrm{~A}\end{array}$ & $\begin{array}{c}0,10 \pm 0,13 \\
\mathrm{~A}\end{array}$ \\
\hline \multicolumn{10}{|c|}{ Linfócitos $\left(\mathrm{x} 10^{3} / \mu \mathrm{L}\right)$} \\
\hline & $\begin{array}{c}3,15 \pm 1,00 \\
A\end{array}$ & $\begin{array}{c}2,48 \pm 0,98 \\
\mathrm{~A}\end{array}$ & $\begin{array}{c}2,79 \pm 1,35 \\
\mathrm{~A}\end{array}$ & $\begin{array}{c}2,16 \pm 0,53 \\
A\end{array}$ & $\begin{array}{c}2,12 \pm 0,60 \\
\mathrm{~A}\end{array}$ & $\begin{array}{c}2,36 \pm 0,46 \\
\mathrm{~A}\end{array}$ & $\begin{array}{c}2,93 \pm 1,36 \\
\mathrm{~A}\end{array}$ & $\begin{array}{c}2,19 \pm 0,53 \\
\mathrm{~A}\end{array}$ & $\begin{array}{c}1,93 \pm 1,08 \\
\mathrm{~A}\end{array}$ \\
\hline II & $\begin{array}{c}3,48 \pm 1,09 \\
\mathrm{~A}\end{array}$ & $\begin{array}{c}2,23 \pm 0,80 \\
\mathrm{~A}\end{array}$ & $\underset{\mathrm{A}}{2,23 \pm 1,01}$ & $\begin{array}{c}2,30 \pm 0,96 \\
\mathrm{~A}\end{array}$ & $\begin{array}{c}1,46 \pm 0,65 \\
\mathrm{~A}\end{array}$ & $\underset{\mathrm{B}}{1,11 \pm 0,65}$ & $\begin{array}{c}2,05 \pm 0,64 \\
\mathrm{~A}\end{array}$ & $\begin{array}{c}3,02 \pm 0,85 \\
\mathrm{~A}\end{array}$ & $\begin{array}{c}3,13 \pm 1,62 \\
\mathrm{~A}\end{array}$ \\
\hline II & $3,10 \pm 0,92$ & $2,19 \pm 0,76$ & $2,09 \pm 0,83$ & $1,95 \pm 0,67$ & $1,43 \pm 0,66$ & $1,22 \pm 0,74$ & $2,00 \pm 0,55$ & $2,94 \pm 0,86$ & $2,66 \pm 0,38$ \\
\hline & & & & $\mathrm{A}$ & $\mathrm{A}$ & & & & \\
\hline IV & $\begin{array}{c}3,25 \pm 1,14 \\
\mathrm{~A}\end{array}$ & $\begin{array}{c}35 \pm 1,27 \\
\mathrm{~A}\end{array}$ & $\begin{array}{c}, 59 \pm 0,88 \\
\mathrm{~A}\end{array}$ & $\begin{array}{c}2,33 \pm 0,98 \\
\mathrm{~A}\end{array}$ & $\begin{array}{c}1,80 \pm 0,94 \\
\mathrm{~A}\end{array}$ & $\begin{array}{c}1,81 \pm 0,84 \\
\mathrm{AB}\end{array}$ & $\begin{array}{c}2,52 \pm 0,98 \\
\mathrm{~A}\end{array}$ & $\begin{array}{c}2,75 \pm 1,05 \\
\mathrm{~A}\end{array}$ & $\begin{array}{c}2,93 \pm 1,12 \\
\mathrm{~A}\end{array}$ \\
\hline \multicolumn{10}{|c|}{ Basófilos $\left(\times 10^{3} / \mu \mathrm{L}\right)$} \\
\hline & $\begin{array}{c}0,09 \pm 0,01 \\
\mathrm{~A}\end{array}$ & $\begin{array}{c}0,09 \pm 0,04 \\
\mathrm{~A}\end{array}$ & $\begin{array}{r}0,03 \pm 0 \\
\mathrm{~A}\end{array}$ & $\begin{array}{c}0,08 \pm 0,23 \\
\mathrm{~A}\end{array}$ & $\begin{array}{c}0,13 \pm 0,13 \\
\mathrm{~A}\end{array}$ & $\begin{array}{r}0,03 \pm 0 \\
\mathrm{~A}\end{array}$ & $\begin{array}{r}0,04 \pm \\
A\end{array}$ & $\begin{array}{r}0,00 \pm \\
\mathrm{A}\end{array}$ & $\begin{array}{c}0,00 \pm 0,00 \\
\text { B }\end{array}$ \\
\hline II & $\begin{array}{c}0,04 \pm 0,05 \\
\mathrm{~A}\end{array}$ & $\frac{0,12 \pm 0,10}{\mathrm{~A}}$ & $\begin{array}{c}0,05 \pm 0,05 \\
\mathrm{~A}\end{array}$ & $\begin{array}{c}0,02 \pm 0,03 \\
\mathrm{~A}\end{array}$ & $\begin{array}{c}0,02 \pm 0,04 \\
\mathrm{~A}\end{array}$ & $\begin{array}{r}0,00 \pm 0 \\
\mathrm{~A}\end{array}$ & $\begin{array}{c}0,09 \pm 0, \\
\mathrm{~A}\end{array}$ & $\begin{array}{c}0,06 \pm 0,06 \\
\mathrm{~A}\end{array}$ & $\begin{array}{c}0,09 \pm 0,08 \\
\mathrm{AB}\end{array}$ \\
\hline III & $\begin{array}{c}0,08 \pm 0,07 \\
\text { A }\end{array}$ & $\begin{array}{c}0,02 \pm 0,03 \\
\mathrm{~A}\end{array}$ & $\begin{array}{c}0,05 \pm 0,07 \\
\text { A }\end{array}$ & $\begin{array}{c}0,04 \pm 0,07 \\
\text { A }\end{array}$ & $\begin{array}{c}0,07 \pm 0,00 \\
\mathrm{~A}\end{array}$ & $\underset{\mathrm{A}}{0,05 \pm 0,00}$ & $\underset{\mathrm{A}}{0,06 \pm 0,07}$ & $\underset{\mathrm{A}}{0,11 \pm 0,11}$ & $\underset{\mathrm{A}}{0,15 \pm 0,13}$ \\
\hline IV & $\begin{array}{c}0,10 \pm 0,12 \\
\mathrm{~A}\end{array}$ & $\begin{array}{c}0,08 \pm 0,02 \\
\text { A }\end{array}$ & $\begin{array}{c}0,07 \pm 0,06 \\
\text { A }\end{array}$ & $\begin{array}{c}0,08 \pm 0,09 \\
\text { A }\end{array}$ & $\begin{array}{c}0,08 \pm 0,14 \\
\text { A }\end{array}$ & $\begin{array}{c}0,04 \pm 0,05 \\
\text { A }\end{array}$ & $\begin{array}{c}0,03 \pm 0,06 \\
\mathrm{~A}\end{array}$ & $\begin{array}{c}0,10 \pm 0,12 \\
\mathrm{~A}\end{array}$ & $\begin{array}{c}0,07 \pm 0,07 \\
\mathrm{AB}\end{array}$ \\
\hline \multicolumn{10}{|c|}{ Eosinófilos $\left(\mathrm{x} 10^{3} / \mu \mathrm{L}\right)$} \\
\hline & $\begin{array}{c}0,13 \pm 0,05 \\
\mathrm{~A}\end{array}$ & $\begin{array}{c}0,28 \pm 0,14 \\
\mathrm{~A}\end{array}$ & $\begin{array}{c}0,22 \pm 0,24 \\
\mathrm{~A}\end{array}$ & $\begin{array}{c}0,21 \pm 0,15 \\
\mathrm{~A}\end{array}$ & $\begin{array}{c}0,20 \pm 0,15 \\
\mathrm{~A}\end{array}$ & $\begin{array}{c}0,12 \pm 0,09 \\
\text { A }\end{array}$ & $\begin{array}{r}0,52 \pm 0 \\
\mathrm{~A}\end{array}$ & $\begin{array}{c}0,14 \pm 0 \\
\mathrm{~A}\end{array}$ & $\begin{array}{c}0,28 \pm 0,14 \\
\mathrm{~A}\end{array}$ \\
\hline II & $\begin{array}{c}0,24 \pm 0,24 \\
\mathrm{~A}\end{array}$ & $\begin{array}{c}0,34 \pm 0,27 \\
\mathrm{~A}\end{array}$ & $\underset{\mathrm{A}}{0,17 \pm 0,14}$ & $\underset{\mathrm{A}}{0,13 \pm 0,14}$ & $\underset{\mathrm{B}}{0,02 \pm 0,04}$ & $\begin{array}{c}0,08 \pm 0,02 \\
\mathrm{~A}\end{array}$ & $\begin{array}{c}0,17 \pm 0,12 \\
\mathrm{~A}\end{array}$ & $\begin{array}{c}0,21 \pm 0,19 \\
\mathrm{~A}\end{array}$ & $\frac{0,22 \pm 0,07}{\mathrm{~A}}$ \\
\hline Ш & $\begin{array}{c}0,42 \pm 0,04 \\
\mathrm{~A}\end{array}$ & $\begin{array}{c}0,30 \pm 0,24 \\
\mathrm{~A}\end{array}$ & $\begin{array}{c}0,28 \pm 0,25 \\
\mathrm{~A}\end{array}$ & $\begin{array}{c}0,24 \pm 0,15 \\
\mathrm{~A}\end{array}$ & $\begin{array}{c}0,21 \pm 0,28 \\
\mathrm{~A}\end{array}$ & $\begin{array}{c}0,17 \pm 0,07 \\
\mathrm{~A}\end{array}$ & $\begin{array}{c}0,18 \pm 0,09 \\
\mathrm{~A}\end{array}$ & $\begin{array}{c}0,26 \pm 0,36 \\
\mathrm{~A}\end{array}$ & $\begin{array}{c}0,21 \pm 0,11 \\
\mathrm{~A}\end{array}$ \\
\hline IV & $\begin{array}{c}0,14 \pm 0,21 \\
\mathrm{~A}\end{array}$ & $\begin{array}{c}0,20 \pm 0,21 \\
\mathrm{~A}\end{array}$ & $\begin{array}{c}0,23 \pm 0,13 \\
\mathrm{~A}\end{array}$ & $\begin{array}{c}0,29 \pm 0,25 \\
\mathrm{~A}\end{array}$ & $\begin{array}{c}0,05 \pm 0,08 \\
\mathrm{~B}\end{array}$ & $0,07 \pm 0,17$ & $0,30 \pm 0,25$ & $\begin{array}{c}0,20 \pm 0,23 \\
\mathrm{~A}\end{array}$ & $\begin{array}{c}0,12 \pm 0,19 \\
\mathrm{~A}\end{array}$ \\
\hline \multicolumn{10}{|c|}{ Monócitos $\left(\mathrm{x} 10^{3} / \mu \mathrm{L}\right)$} \\
\hline & $\begin{array}{c}0,08 \pm 0,06 \\
\mathrm{~A}\end{array}$ & $\begin{array}{c}0,19 \pm 0,02 \\
\mathrm{~A}\end{array}$ & $\begin{array}{c}0,27 \pm 0,15 \\
\mathrm{~A}\end{array}$ & $\begin{array}{c}0,20 \pm 0,04 \\
\text { A }\end{array}$ & $\begin{array}{c}0,28 \pm 0,17 \\
\mathrm{~A}\end{array}$ & $\begin{array}{r}0,20 \pm 0 \\
\mathrm{~A}\end{array}$ & $\begin{array}{c}0,22 \pm 0,07 \\
\text { B }\end{array}$ & $\begin{array}{c}0,23 \pm 0,08 \\
\mathrm{~B}\end{array}$ & $\begin{array}{c}0,20 \pm 0,13 \\
\mathrm{~A}\end{array}$ \\
\hline II & $\begin{array}{c}0,12 \pm 0,05 \\
\mathrm{~A}\end{array}$ & $\begin{array}{c}0,09 \pm 0,03 \\
\mathrm{~A}\end{array}$ & $\begin{array}{c}0,21 \pm 0,15 \\
\mathrm{~A}\end{array}$ & $\begin{array}{c}0,19 \pm 0,12 \\
\mathrm{~A}\end{array}$ & $\begin{array}{c}0,15 \pm 0,11 \\
\mathrm{~A}\end{array}$ & $\begin{array}{c}0,45 \pm 0,07 \\
\mathrm{~A}\end{array}$ & $\begin{array}{c}0,56 \pm 0,22 \\
\text { A }\end{array}$ & $\begin{array}{c}0,30 \pm 0,23 \\
\mathrm{~A}\end{array}$ & $\begin{array}{c}0,27 \pm 0,16 \\
\mathrm{~A}\end{array}$ \\
\hline III & $\underset{\mathrm{A}}{0,16 \pm 0,11}$ & $\begin{array}{c}0,08 \pm 0,06 \\
\mathrm{~A}\end{array}$ & $\begin{array}{c}0,14 \pm 0,11 \\
\mathrm{~A}\end{array}$ & $\begin{array}{c}0,14 \pm 0,09 \\
\mathrm{~A}\end{array}$ & $\begin{array}{c}0,20 \pm 0,14 \\
\mathrm{~A}\end{array}$ & $\underset{\mathrm{A}}{0,44 \pm 0,10}$ & $0,37 \pm 0,27$ & $\begin{array}{c}0,16 \pm 0,10 \\
\mathrm{~A}\end{array}$ & $\underset{\mathrm{A}}{0,23 \pm 0,15}$ \\
\hline V & $\begin{array}{c}0,19 \pm 0,11 \\
\mathrm{~A}\end{array}$ & $\begin{array}{c}0,11 \pm 0,06 \\
\mathrm{~A}\end{array}$ & $\begin{array}{c}0,25 \pm 0,21 \\
\mathrm{~A}\end{array}$ & $\begin{array}{c}0,19 \pm 0,13 \\
\mathrm{~A}\end{array}$ & $\begin{array}{c}0,13 \pm 0,15 \\
\mathrm{~A}\end{array}$ & $\begin{array}{c}0,39 \pm 0,34 \\
\mathrm{~A}\end{array}$ & $\begin{array}{c}0,27 \pm 0,12 \\
\mathrm{AB}\end{array}$ & $\begin{array}{c}0,21 \pm 0,17 \\
\mathrm{~A}\end{array}$ & $\begin{array}{c}0,27 \pm 0,12 \\
\mathrm{~A}\end{array}$ \\
\hline
\end{tabular}

T0: basal; T1i-T3i: horas correspondentes à fase de isquemia; T3r-T168r: horas correspondentes à fase de reperfusão ou de desobstrução; $\mathrm{I}=$ grupo controle (GI); II = grupo de animais submetidos a isquemia e reperfusão do duodeno (GII); III = animais submetidos a isquemia e reperfusão do íleo (GIII); IV = animais submetidos a isquemia e reperfusão do cólon maior (GIV).

Médias seguidas por letras distintas, na mesma coluna, diferem entre si pelo teste de Tukey, no nível de significância de 5\%, e estabelecem comparação entre os diferentes grupos em cada momento. 
Ainda no T72r, nos animais do grupo GII, houve aumento no número de monócitos. Monocitose pode ocorrer sempre que houver necrose tecidual e demanda de fagócitos, ou seja, quadros de necrose, supuração e trauma, como ressaltado por ZINKL (1989).

No final do período de observação (T168r), os equinos do grupo GIII apresentaram aumento do número de basófilos. Aumento que justifica o papel patofisiológico dos basófilos na indução da inflamação, da coagulação e da fibrinólise, por meio das propriedades biológicas dos fatores presentes em seus grânulos e, também, nas fases mais tardias das reações inflamatórias, como citado por JAIN (1993).

Diferindo dos resultados obtidos por FAGLIARI et al. (2008) em equinos com cólica submetidos à laparotomia - nos quais os maiores percentuais de elevação das contagens de leucócitos, especialmente de polimorfonucleares e de bastonetes deveram-se, possivelmente, a distúrbios inflamatórios e infecciosos decorrentes do choque séptico -, pode-se, considerar que os resultados do leucograma apresentados servem como referência para equinos com cólica submetidos a laparotomia, cujo pós-operatório evolui sem intercorrência. Desse modo, uma cinética da resposta leucocitária diferente da apresentada pode indicar a presença de foco infeccioso durante o pós-operatório ou agravamento do quadro clínico.

\section{CONCLUSÃO}

Nas condições em que este experimento foi realizado apenas os equinos com obstrução de duodeno e de íleo apresentaram, como consequência da lesão entérica, alterações clínicas e hematológicas significativas. Esse fato indica que algumas alterações no eritroleucograma, quando analisadas em conjunto com os dados obtidos no exame físico, podem auxiliar na identificação do segmento intestinal obstruído, na elaboração do prognóstico e no acompanhamento da evolução do processo de cura.

\section{AGRADECIMENTO}

À Fundação de Amparo à Pesquisa do Estado de São Paulo (Fapesp) pelo financiamento integral desta pesquisa (processos no 05/58712-0 e 06/55377-8).

COMITÊ DE ÉTICA E BIOSSEGURANÇA

Aprovado pela Comissão de Ética e Bem-Estar Animal (CEBEA), protocolo 꿍 023232-05.

\section{REFERÊNCIAS}

BASILE-FILHO, A.; CUNHA, F. Q.; TAVARES-MURTA, B. M.; MARTINS, M. A.; ARRAES, S. M. A. A. Falência de migração de neutrófilos e dosagem sérica de citocinas e óxido nítrico na sepse humana. Revista Brasileira de Terapia Intensiva, v. 14, n. 4, p. 146-155, 2002.

DATT, S. C.; USENIK, E. A. Intestinal obstruction in the horse: physical signs and blood chemistry. The Cornell Veterinárian, v. 65, n. 2 , p. $152-172,1975$.

DI FILIPPO, P. A.; SANTANA, A. E.; PEREIRA, G. T. Equilíbrio ácido-base e hidroeletrolítico em equinos com cólica. Ciência Rural, v. 38, n. 4, p. 1.003-1009, 2008

FAGLIARI, J. J.; SILVA, S. L. Hemograma e proteinograma plasmático de equinos hígidos e de equinos acometidos por abdômen agudo, antes e após laparotomia. Arquivo Brasileiro de Medicina Veterinária e Zootecnia, v. 54, n. 6, p. 559-567, 2002.

FAGLIARI, J. J.; SILVA, S. L.; SILVA, P. C.; PEREIRA, G. T. Leucograma e teores plasmáticos de proteínas de fase aguda de equinos portadores de abdômen agudo e submetidos à laparotomia. Arquivo Brasileiro de Medicina Veterinária e Zootecnia, v. 60, n. 2, p. 322-328, 2008.

FALEIROS, R. R.; ALVES, G. E. S.; SANTOS, R. L.; MARQUES JR., A. P.; MACORIS, D. G. Experimental ischemia and reperfusion in equine small colon. Arquivo Brasileiro de Medicina Veterinária e Zootecnia, v. 53, n. 3, p. 341-350, 2001.

JAIN, N. C. Essencials of veterinary hematology. Philadelphia: Lea \& Febiger, 1993. 417 p.

LASSEN, E. D.; SWARDSON, C. J. Hematology and hemostasis in the horse: normal functions and common abnormalities. Veterinary Clinics of North America. Equine practice, v. 11, n. 3, p. 351-389, 1995.

MONTELlO, T. G.; CASTRO JR., J. F. C.; SANTOS, V. P.; CHRISTO, E. C. S.; FILHO, A. P. F. S. Alterações hematológicas observadas em equinos submetidos à laparotomia em estação e enterotomia do cólon menor. Acta Scientiae Veterináriae, v. 32, n. 3, p. 201-205, 2004.

MOORE, J. M.; BARTON, M. H. Treatment of endotoxemia. Veterinary Clinics of North America. Equine Practice, v. 19, n. 2, p. 681-695, 2003.

NATALINI, C. C.; ROBINSON, E. P. Evaluation of analgesic effects of epidurally administered morphine, alfentanil, butorphanol, tramadol and U50488H in horses. American Journal of Veterinary Research, v. 61, n. 12, p. 1.576-1.586, 2000. 
PUOTUNEN-REINERT, A.; HUSKAMP, B. Experimental duodenal obstruction in the horse. Veterinary Surgery, v. 15, n. 6 , p. 420-428, 1986.

SAMPAIO, I. B. M. Estatística aplicada à experimentação animal. 2. ed. Belo Horizonte: Fundação de Estudos e Pesquisas em Medicina Veterinária e Zootecnia, 2002. 265 p.

SOUTHWOOD, L. Acute abdomen. Veterinary Clinics of North America. Equine Practice, v. 5, n. 2, p. 112-126. 2006.

SPEIRS, C. V. Clínical examination of horses. Pennsylvania: Saunders Company, 1997. 358 p.
SVENDSEN, C. K.; HJORTKJAER, R. K.; HESSELHOLT, M. Colic in the horse: a Clínical and Clínical chemical study of 42 cases. Nordisk Veterinaermedicin, v. 31, n. 10, p. 1-32, 1979.

TURNER, A. S.; McILWRAITH, C. W. Laparotomia do flanco e exploração abdominal. In: Técnicas cirúrgicas em animais de grande porte. São Paulo: Roca, 2002. p. 237-242.

ZINKL, J. G. Leukocyte function. In: KANEKO, J. J. Clínical biochemistry of domestic animals. 4. ed. San Diego: Academic Press, 1989. p. 316-334.

Protocolado em: 23 set. 2008. Aceito em: $1^{\circ}$ dez. 2010. 